\title{
De la calle al parlamento: trayectorias y repertorios de una generación de estudiantes. Chile, 2006-2017 ${ }^{1}$
}

\author{
Marion Di Méo²
}

Fecha de recepción: 12 de marzo de 2019

Fecha de aprobación: 6 de mayo de 2019

Fecha de publicación: 30 de julio de 2019

\begin{abstract}
Resumen
Más de quince años después de la transición a la democracia, los estudiantes chilenos protagonizaron dos ciclos de movilizaciones sociales, sin precedentes desde las protestas nacionales (1983-1986) en contra del régimen autoritario de A. Pinochet. Este artículo se propone examinar estos ciclos, con especial atención a la cuestión generacional y sus implicaciones en el fomento de un movimiento social que tendrá un impacto importante, tanto en la agenda pública nacional como en la vida política del país. Se tratará también de analizar el repertorio de la acción colectiva de este movimiento heterogéneo, revelando así su adaptabilidad estratégica y sus divisiones internas. Finalmente, el artículo presta atención a los fenómenos de reconversión de algunos dirigentes estudiantiles a la política institucional.
\end{abstract}

Palabras clave: movilizaciones estudiantiles, generaciones, politización, repertorio

\section{From the streets to the parliament: trajectories and repertoires of a student generation.}

Chile, 2006-2017

\begin{abstract}
More than fifteen years after the transition to democracy, Chilean students staged two cycles of social mobilization, unprecedented since the national protests (1983-1986) against the authoritarian regime of Augusto Pinochet. This article intends to examine these cycles with special attention to the generational issue and its implications on the consolidation of a social
\end{abstract}

Este artículo presenta algunas de las conclusiones de la tesis doctoral de la autora: "Una democracia a prueba de los movimientos sociales: el caso del Chile post-dictatorial de 1988 a la actualidad", defendida públicamente en noviembre de 2018 en la Universidad de Aix-Marseille (Francia) y financiada por dicha institución. 2 Doctora en Ciencia Política por la Universidad de Aix-Marseille, Francia. CHERPA-Instituto de Estudios Políticos de Aix-en-Provence, Francia. Contacto: marion.dimeo@hotmail.fr 
De la calle al parlamento: trayectorias y repertorios de una generación de estudiantes. Chile, 2006-2017 -

movement that will have a large impact on both the national public agenda and the political life of the country. It also looks to analyze the repertoire of collective action of this heterogeneous movement, revealing its strategic adaptability and its internal divisions. Finally, this paper pays attention to the phenomena in which some student leaders are reconverted to institutional politics.

Keywords: student protests, generations, politicization, repertoire

\title{
Da rua ao parlamento: trajetórias e repertórios de uma geração de estudantes. Chile, 2006-2017
}

\begin{abstract}
Resumo
Mais de quinze anos após a transição à democracia, os estudantes chilenos protagonizaram dois ciclos de mobilizações sociais, sem precedentes desde os protestos nacionais (1983-1986) contra o regime autoritário de A. Pinochet. Este artigo propõe-se examinar estes ciclos, com especial atenção à questão geracional e suas implicações no fomento de um movimento social que irá ter um impacto importante, tanto na agenda pública nacional quanto na vida política do país. Tenta, também, analisar o repertório da ação coletiva deste movimento heterogêneo, revelando assim sua adaptabilidade estratégica e suas divisões internas. Finalmente, o artigo presta atenção aos fenômenos de reconversão de alguns dirigentes estudantis à política institucional.
\end{abstract}

Palavras-chave: mobilizações estudantis, gerações, politização, repertório

\section{Introducción: protestar en el Chile posdictatorial}

Santiago de Chile, julio del 2011: miles de estudiantes salieron a las calles del país para pedir reformas estructurales del sistema educativo, denunciando las consecuencias de dicho sistema y ofreciendo alternativas y propuestas concretas para reformar la educación chilena. Esas protestas masivas, que ocurrieron poco después de las de organizaciones ecologistas en contra de un megaproyecto energético entre enero y marzo, fueron apoyadas por otros sectores de la sociedad que se movilizaron junto a los estudiantes - docentes, algunos rectores de universidades y los trabajadores de la Central Unitaria de Trabajadores (CUT), que convocó a dos días de paro nacional el 24 y 25 de agosto. Las protestas de 2011 cuestionaron con fuerza tanto el modelo educativo vigente como las instituciones políticas del país, demostrando a 
la vez el afán de participación y las capacidades organizacionales y de movilización de la juventud de Chile.

Sin embargo, esas acciones colectivas contenciosas están lejos de ser evidentes en un país caracterizado por diecisiete años de dictadura. En los años 1990, Chile entra en un "ciclo recesivo de movilización" (Barozet, 2011a, p. 73), mientras que se pone en marcha una democracia designada por distintos calificativos, tales como "de baja intensidad" (Moulián, 1997), "semi-soberana" (Huneeus, 2014) o "incompleta" (Garretón y Garretón, 2010), que tienden a subrayar la persistencia de mecanismos institucionales y prácticas políticas que obstaculizan el sistema político ${ }^{3}$. Tomas Moulián (1997) subraya que los gobiernos de la Concertación ${ }^{4}$ tienden a desalentar la participación ciudadana y la movilización social en nombre de la estabilidad política y del crecimiento económico. Después de la transición, los nuevos gobiernos heredan un sistema político marcado por la persistencia de enclaves autoritarios (Garretón, 1989), que O. Dabène describe como "salvaguardias creados por los militares para proteger por anticipación tanto intereses como orientaciones políticas" (2008, p. 90) 5 . Si muchos de esos enclaves fueron reformados hoy en día, la Constitución aprobada en dictadura (1980) sigue vigente, cuyo sello autoritario representa el principal enclave institucional que permanece hoy en día en Chile.

\footnotetext{
Sobre esos mecanismos, Carlos Huneeus menciona por ejemplo lo que llama el "poder de veto de la minoría", la continuidad del general Pinochet y su participación política, o algunos dominios reservados de los militares. Evoca también la falta de confianza en las instituciones políticas y un respaldo a la democracia bastante inferior a los niveles de otros países latinoamericanos, entre otros.

4 La Concertación de Partidos por la Democracia es una coalición de partidos de centro-izquierda que nace durante la campaña del "No" del plebiscito organizado por el régimen militar en 1988, sobre el mantenimiento o no del general Pinochet a la cabeza del país por ocho años más. Esa coalición agrupa al Partido socialista, el Partido Demócrata Cristiano, el Partido por la Democracia y otras pequeñas formaciones de izquierda.

5 Entre esos enclaves autoritarios podemos mencionar el hecho de que el general Pinochet se mantiene como comandante en jefe de las Fuerzas Armadas hasta 1998, la existencia de un Consejo de Seguridad Nacional, conformado por miembros de las distintas ramas militares, el sistema electoral binominal, criticado por permitir una sobrerrepresentación de la oposición, o la existencia de senadores vitalicios.
} 
Esos dilemas político-institucionales existen junto a enclaves más simbólicos y culturales, analizados en detalle por Doran (2016). Demuestran la manera con la que se construye, en los discursos políticos oficiales, en particular presidenciales, una imagen negativa de la acción colectiva, que se asocia a la idea de "conflicto" y de amenaza a la nueva convivencia. Se desactivan los actores sociales que se movilizaron durante la dictadura, en un contexto de "aplicación elitista del funcionamiento de la democracia” (Barozet, 2011a).

A esos elementos institucionales, políticos y simbólicos, hay que agregar una dimensión económica y recordar que los gobiernos de la Concertación tomaron la decisión de seguir con las características generales del modelo neoliberal establecido por el régimen autoritario (Garretón, 2010; Gaudichaud, 2014). La diferencia principal con el periodo dictatorial fue el aumento del gasto público en políticas sociales, que permitieron reducir la pobreza absoluta sin alterar las grandes desigualdades distributivas o territoriales vigentes (Gaudichaud, 2014). El sistema educativo es un caso emblemático de implementación de medidas neoliberales, como lo veremos más adelante.

En ese contexto, poco favorable a la contestación, los estudiantes chilenos protagonizaron dos intensos ciclos de movilizaciones: primero en 2006, luego en 2011. La bibliografía actualmente disponible sobre esos eventos es inmensa y abarca distintas disciplinas de las ciencias sociales, por lo cual resulta imposible atender a las numerosas referencias en el marco de este artículo. De manera general, lo que nos gustaría señalar es que muy pocos estudios (Vera Gajardo, 2011; Donoso, 2016) llevan a cabo una reflexión detallada y sistematizada en torno a los repertorios de la acción colectiva, así como a sus implicaciones para la movilización estudiantil. Existen también muchos estudios que tienen como objeto central las demandas formuladas por los estudiantes en relación al modelo educativo existente (Assael, Cornejo, Gonzalez, Redondo, Sanchez y Sobarz, 2011; Azócar, 2013; Braghetto, 2013) o que analizan al movimiento estudiantil desde la perspec- 
tiva de los ciclos de movilización (Thielemann, 2011; Muñoz Tamayo y Durán, 2019).

El objetivo central de este artículo es dar a entender algunas razones que permiten explicar el porqué de estas protestas, y analizar lo que nos revela el cómo de esas movilizaciones. Defendemos la idea de que un análisis en términos generacionales permite poner de relieve la existencia de procesos de aprendizaje y politización de una generación de estudiantes, claves para llevar a cabo dos ciclos de contestación en contextos políticos diferentes. Gracias al examen de los distintos elementos que componen el "repertorio de acción colectiva" (Tilly, 1984) de los estudiantes chilenos, evidenciaremos tanto los clivajes políticos del movimiento como su capacidad estratégica y de adaptación a las respuestas del Estado y sus propias divisiones. Llevaremos una reflexión un poco más detallada sobre el tema del uso de la violencia, que poco aparece en las investigaciones sobre el repertorio de acción colectiva de los estudiantes, cuando se da a ver en muchas manifestaciones estudiantiles. El examen de los modos de acción colectiva de los estudiantes nos llevará finalmente a analizar la reconversión hacia la política institucional de algunos líderes de las movilizaciones de 2011, así como su impacto sobre el sistema de partidos chilenos que fue parcialmente renovado pocos años después de las protestas.

Los elementos presentados aquí se sustentan primero en la observación de numerosas manifestaciones estudiantiles y performances callejeras que tuvieron lugar en Santiago entre 2011 y $2017^{6}$, y de actividades políticas realizadas en distintas universidades de la capital — asambleas, tomas, debates, eventos culturales-. Se basan también en la realización de treinta y dos entrevistas semidirectivas con militantes y dirigentes del movimiento

Resulta difícil cifrar con exactitud el número de marchas estudiantiles a las que asistimos durante nuestras estancias en Santiago. Diríamos que esta cifra gira en torno a las treinta manifestaciones en Santiago y dos en Valparaíso. 
secundario y universitario durante distintas estancias en Chile ${ }^{7}$, y en la compilación de archivos de prensa sobre las manifestaciones estudiantiles de los años 2006 y $2011^{8}$.

El artículo aborda primero el contexto de ocurrencia de las movilizaciones previamente mencionadas y los elementos que llevaron a la "primavera de Chile" del año 2011, prestando atención especial a los efectos de la renovación generacional en el movimiento estudiantil. En segundo lugar, analizaremos los medios de acción llevados a cabo por los estudiantes, desde la manifestación hasta la barricada, demostrando que sus decisiones dependen tanto de la percepción de los contextos políticos como de las dinámicas internas de este movimiento heterogéneo. Volveremos en una última sección sobre los fenómenos de reconversión de algunos dirigentes estudiantiles.

\section{Contestación del modelo educativo en contexto cambiante y renovación generacional}

Como lo mencionamos brevemente en la introducción, el sistema educativo constituye uno de los sectores más afectados por la implementación de reformas de inspiración neoliberal. Se tomaron las primeras medidas transformación de la educación chilena a mediados de los años 1970, cuando se introdujo un nuevo método de financiamiento de la educación primaria y secundaria. En la primera etapa de la dictadura se observa una intervención política de la educación superior, es decir, se pone fin al autogobierno y a la autonomía de las distintas instituciones uni-

\footnotetext{
La primera estancia se realizó entre julio del 2011 y julio del 2012, durante un intercambio universitario en la Pontificia Universidad Católica de Santiago, en plenas protestas estudiantiles. Las otras estancias, más cortas (tres meses en promedio), se realizaron en 2014 (enero-febrero), 2015 (marzo-junio), 2016 (julio-octubre) y 2017 (abril-mayo).

8 Compilamos una gran cantidad de artículos de prensa de los periódicos $\mathrm{La}$ Nación y La Tercera, que trataban de las movilizaciones estudiantiles del mes de mayo de 2006 y entre mayo y noviembre del 2011.
} 
versitarias, se designan rectores favorables al nuevo régimen y se

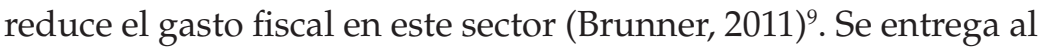
Estado un papel subsidiario en materia de educación (Assael et al., 2011), y el giro más importante en la enseñanza superior se da en 1981, cuando el nuevo marco legislativo implementado por el régimen militar busca "desplazar el centro de gravedad del sistema en dirección de los mercados" (Brunner, 2011, p. 5). Se inicia así un proceso de privatización de la educación terciaria - desmantelamiento de las universidades estatales, reducción drástica de la financiación pública, apoyo a la creación de entidades educativas privadas "casi sin barreras de acceso" (Brunner, 2011, p. 5), entre otros (Assael et al., 2011). Este mismo año, el régimen militar empieza un proceso de descentralización, que consiste en transferir la administración de las escuelas primarias y de los colegios públicos a los municipios. El sistema de financiamiento que se pone en marcha se sustenta en vouchers, que se reparten a los establecimientos escolares según el número de alumnos que reciben. El 10 de marzo de 1990, la Ley Orgánica Constitucional de Educación (LOCE) ratifica e institucionaliza esos cambios, y el estatuto especial de esta ley hace que cualquier intento de modificación o abrogación debe ser aprobado por altísimas mayorías en la Cámara de Diputados y en el Senado.

Con la vuelta a la democracia, los nuevos gobiernos democráticos decidieron aumentar el gasto público en el sector educativo - de un 2,6\% a un 4,3\% del PIB entre 1990 y 2000, cifra que sigue subiendo en el 2000 (Muñoz y Raczynski, 2007)—, dar prioridad al alza de la cobertura escolar y mejorar las condiciones laborales de los profesores - promulgación del estatuto docente para el personal municipal, aumento de los sueldos. Mejoras en materia de infraestructuras — bibliotecas, salas informáticas- y

La educación superior en Chile está compuesta por tres tipos de establecimientos, según el nivel y el tipo de programas ofrecidos: las universidades, los institutos profesionales - que entregan diplomas profesionales sin exigir la licenciatura previa-y los centros de formación técnica (Brunner, 2009). 
la instauración de la jornada escolar completa son algunos de los logros de la Concertación que cabe mencionar en materia de educación (Águila, 2010). A pesar de esos esfuerzos, Chile sigue siendo uno de los países de la Organización de Cooperación y Desarrollo Económico (OCDE) que menos invierte en educación $^{10}$. El fin de la gratuidad de la educación superior y el sistema de autofinanciamiento de las universidades generaron un alza importante de la matrícula y la educación chilena es de las más caras del mundo —alrededor del 22\% del PIB per capita-, costo que recae principalmente en los alumnos y sus familias. Parte creciente del acceso a la educación superior se financia mediante la lógica del endeudamiento, con tasas de interés que alcanzan hasta un $8 \%$ de interés según los bancos (Braghetto, 2013). El sistema de financiamiento, así como la autonomía de algunos establecimientos escolares, permite la existencia de procesos lucrativos —lo que los chilenos designan como "lucro"—, denunciado en múltiples ocasiones por los estudiantes y sus familiares. Las distintas movilizaciones de los jóvenes chilenos desde el retorno a la democracia giraron en torno a estas consecuencias del modelo educativo, y no fue hasta el año 2016 que se introdujeron cambios significativos al sistema. Aunque todavía sea pronto para evaluar el impacto de estas reformas, llevadas a cabo por el segundo gobierno de Michelle Bachelet (2014-2018), podemos mencionar la aprobación en marzo de 2016, de la "Ley de Inclusión", que plantea, entre otras cosas, el término de las practicas lucrativas en los establecimientos secundarios, con la obligación por los administradores de reinvertir todos los fondos públicos recibidos durante el curso del año; o el fin de la selección de los alumnos sobre la base de criterios socioeconómicos ${ }^{11}$. Cabe desta-

\footnotetext{
10 Ver http://www.cooperativa.cl/noticias/sociedad/educacion/ocde-chile-esel-pais-con-menor-gasto-publico-en-educacion/2013-10-19/200351.html.

11 «Ley de inclusión escolar : por un país con mejor integridad social», Agencia de Calidad de la Educación. URL: http://www.agenciaeducacion.cl/noticias/leyinclusion-escolar-pais-mayor-integridad-social/. Consultado el 15 de marzo del 2019.
} 
car la promulgación, en noviembre de 2017, de la ley de "Nueva Educación Pública", que organiza la transferencia de la administración de los colegios públicos, hasta ahora gestionados por las municipalidades, hacia organismos directamente vinculados al Ministerio de Educación. Por último mencionaremos el voto, en enero de 2018, de una ley que rige las modalidades de financiamiento de la educación superior. Este texto introduce el principio de gratuidad de las universidades estatales para el 60\% de los alumnos $^{12}$.

Estas reformas fueron en gran parte posibles por la movilización colectiva de una nueva generación de estudiantes que cuestionaron con fuerza el sistema educativo. Sin embargo, las protestas estudiantiles de los años 1990 ya se oponen al proceso de mercantilización de la educación pública, y "parecen romper con el inmovilismo social al cual la sociedad parecía condenada" (Cortés, 2015, p. 20). Los estudiantes piden solución al problema del financiamiento, el aumento de la ayuda del Estado (Avendaño, 2014, p. 47) y se oponen a la liquidación definitiva de las universidades públicas. Las manifestaciones del año 1992 en contra de la privatización de la Universidad de Santiago (USACH), y luego las de 1997 en reacción a la "Ley Marco de las Universidades Estatales", que ratifica el autofinanciamiento de dichos establecimientos, van esa dirección. Dos elementos sin embargo tienden a generar actitudes de contención de la contestación universitaria. Se trata primero de la postura poco conciliadora de los estudiantes afiliados a las juventudes de los partidos de la Concertación hacia la movilización. Por otro lado, la participación de antiguos líderes estudiantiles en el gobierno de Ricardo Lagos alienta este proceso. La prioridad de los estudiantes chilenos fue entonces la de la reconstrucción interna, como lo muestran

12 «Congreso de Chile aprueba la ley de gratuidad de la educación superior», Agencia EFE, 25 de enero de 2018. Consultado el 15 de marzo del 2019. URL: https://www.efe.com/efe/america/sociedad/el-congreso-de-chile-aprueba-laley-gratuidad-educacion-superior/20000013-3503080\#. 
distintos estudios realizados por historiadores tales como Thielemann (2011). Donoso (2016) subraya al respecto: "conscientes del despertar de su capacidad de movilización, los estudiantes hacen de la consolidación de nuevas organizaciones y de una agenda nueva una prioridad" (p. 56). Este proceso lleva al nacimiento de nuevas organizaciones universitarias, tales como la SurDa y la Unión Nacional de los Estudiantes (UNE), mientras que la Federación de Estudiantes de la Universidad de Chile (FECH), dirigida por las juventudes comunistas a partir de 1995, decide volver a dinamizar la Confederación de Estudiantes de Chile $(\mathrm{CONFECH})^{13}$. La reconstrucción del movimiento secundario pasa por la creación de la Asamblea de Coordinación de los Estudiantes Secundarios (ACES), a fines de los 90, instancia que agrupa los centros de alumnos poco inclinados a apoyar a la Concertación. Las primeras reivindicaciones de esta nueva organización son muy concretas y, como lo muestra Donoso (2016), permiten construir una movilización basada en la politización de la experiencia diaria de los alumnos. Hay que subrayar también que la ACES valora estrategias de organización y relaciones entre la base y el liderazgo más "horizontales" — figuras de voceros en vez de presidentes, toma de decisión sometida a la lógica de la asamblea y en continua consulta con sus bases (Muñoz Tamayo y Durán, 2019) - que contrastan con la estructura de otras organizaciones secundarias como la ACAS (Asamblea de los Centros de Alumnos de Santiago) o la FESES (Federación de Estudiantes de Santiago), más piramidales y jerarquizadas (Donoso, 2016; Thielemann, 2011). Hay que tener esos elementos en mente a la hora de analizar el mochilazo de 2001, primera movilización de

13 La CONFECH es una organización estudiantil que agrupa las federaciones de estudiantes de algunas universidades chilenas. Creada en 1984, se compone hoy de las federaciones de las universidades estatales (tales como la Universidad de Chile, la Universidad de Santiago - USACH - o la Universidad Metropolitana de Ciencias de la Educación), de las universidades privadas tradicionales (creadas antes del decreto de 1981 sobre la educación superior, entre las cuales las distintas universidades católicas) y desde del 2011 de algunas universidades privadas (Alberto Hurtado, Diego Portales, Adolfo Ibañez). 
los secundarios desde el final de la dictadura. El alza del precio del pase escolar lleva la ACES a llamar a la movilización y varias manifestaciones, cuya participación varía entre cinco y ocho mil personas, se suceden durante sucesivas semanas, demostrando el potencial movilizador de esta organización y de una nueva generación de jóvenes chilenos. Muñoz Tamayo y Durán (2019) subrayan que el mochilazo introduce "cambios en la cultura política de la movilización secundaria y su modo de entender lo social frente a lo político" (p. 143).

Si varias organizaciones estudiantiles formulan las primeras críticas hacia el modelo educativo en su conjunto desde mediados de los 90, las principales reivindicaciones están principalmente vinculadas a demandas materiales concretas y gremiales (Avendaño, 2014) y las acciones del movimiento son principalmente "defensivas", para detener privatizaciones, por ejemplo, y "paliativas" (Muñoz Tamayo y Durán, 2019, p. 143). Esta situación cambia a mediados de los años 2000, cuando los secundarios salen de nuevo a las calles de Chile, antes de paralizar la actividad de gran parte de los colegios públicos y subvencionados del país durante la "revolución pingüina" y formular una crítica del sistema de educación en su conjunto. Ese cambio de paradigma reivindicativo sienta las bases de las protestas de 2011, cuando estudiantes secundarios y universitarios luchan juntos en un ciclo de protestas sin precedentes en la historia reciente del país.

Así, en 2006, los secundarios están al centro de una importante movilización, cuando ya ha pasado una generación entera en las aulas de los colegios desde el final de la dictadura, sin que se satisficieran plenamente las expectativas de movilidad social de las familias chilenas. La revuelta de los pingüinos empieza en los inicios del primer gobierno de Michelle Bachelet con demandas puntuales - tales como la gratuidad de los exámenes de acceso a la universidad-, para transformarse en una crítica profunda a los fundamentos institucionales del sistema educativo (Muñoz Tamayo y Durán, 2019) representado por la 
LOCE. Las movilizaciones toman rápidamente la forma de un paro nacional, y los secundarios piden la desmunicipalización de la educación primaria y secundaria, el fin de la segregación y del lucro. El mes de mayo se caracteriza por la realización de varias manifestaciones, así como por la parálisis de muchos colegios. Donoso (2016) explica: “a finales de mayo del 2006, doscientos mil estudiantes participan en las ocupaciones de colegios en Santiago, y más de ciento veinte mil en el resto del país" (p. 59). Las movilizaciones llevan la presidenta Bachelet a modificar su agenda y negociar un proyecto de reforma educativa con la oposición, adoptado bajo el nombre de Ley General de Educación (LGE), muy por debajo de las expectativas de los estudiantes. El final del conflicto se traduce en la salida de la mesa de las negociaciones de los representantes de los secundarios y de la cooptación de algunos dirigentes, miembros de las juventudes socialistas, por los dirigentes del Partido Socialista de la época. Ese primer movimiento de contestación llevado a cabo por los estudiantes deja una nota muy amarga, y son muchos los que se sienten traicionados por el gobierno. Muchas organizaciones secundarias optan por el conflicto abierto con las instituciones, fenómeno que se observa también en federaciones universitarias, especialmente después de la publicación de la ley del Crédito con Aval del Estado (CAE) en 2005, que introduce un sistema público de financiamiento de los créditos universitarios con una tasa del 5,4\% (Braghetto, 2013). La adopción de dicha medida, bajo la presidencia de un socialista, favorece la implementación de una estrategia de confrontación con el gobierno.

En el año 2011 fueron primero los estudiantes universitarios quienes iniciaron un nuevo ciclo de intensas movilizaciones. Cabe subrayar que no existe una ruptura nítida entre los actores de estos dos ciclos de movilización, más bien una continuidad generacional en torno a dos elementos: primero, muchos de los estudiantes que participan en la contestación de 2011 ya habían actuado en 2006, especialmente en las ocupaciones de sus cole- 
gios. Veinte de los estudiantes que entrevistamos, al igual con quienes habló Donoso (2016), nos hablaron de dicha participación. Por otra parte, los eventos de 2006 y 2011 movilizan una generación conocida en Chile como la de los "hijos de la democracia", nacida durante o poco después del plebiscito de 1988 y criada en un contexto democrático. Tal como lo explica Vera Gajardo (2011), son los estudiantes mismos que se llaman así, en una lógica de ruptura con las generaciones precedentes. En un contexto que sigue marcado por la brutalidad de los años de dictadura, la cuestión de las generaciones aparece ser una entrada muy fecunda para entender las movilizaciones estudiantiles a partir de los años 2000. Mannheim aborda esta problemática en El problema de las generaciones, en el que identifica tres elementos comunes en la generación (Mannheim, 1990). Se trata primero de la situación de generación, que constituye una potencialidad común, un horizonte compartido por un conjunto de personas nacidas en un mismo periodo, pero que no se activa necesariamente. El solo hecho de ser contemporáneos no basta para crear una generación, y el hecho de compartir un horizonte solo puede producir una generación potencial. Se trata entonces de identificar, más allá de la simple situación que resulta del hecho de pertenecer a una misma unidad histórico-social, la participación en un evento destacado, una especie de experiencia fundadora al origen de disposiciones comunes. De ahí viene el segundo elemento que precisa el concepto de generación, el conjunto generacional, que designa a los individuos que comparten efectivamente un destino común. Se trata finalmente de la unidad de generación, que consiste en un grupo concreto adentro de una generación. Varios autores subrayan también el impacto de los eventos políticos en la socialización y en el aprendizaje de los preadultos (Percheron, 1993; Ihl, 2002). En el caso que nos interesa, el hecho de haber sido criado por padres que vivieron la dictadura, pero sin haberla vivido directamente, constituye un elemento central al origen de un sentimiento de pertenencia generacional de los estudiantes chilenos. 
Daniel Grimaldi (2009) reactiva la problemática de las generaciones en sus investigaciones sobre la evolución del socialismo chileno desde el retorno a la democracia. Considera que los chilenos nacidos a finales de los 80 y principios de los 90 comparten un "efecto cohorte", designando "un grupo de personas nacidas en un mismo intervalo de tiempo, que se encuentran en una etapa similar de su vida y que, a medida que envejecen, comparten experiencias similares" (Grimaldi, 2009, p. 4). El autor nos habla también de un "efecto periodo", que se genera a partir de eventos que afectan de manera significativa una sociedad determinada: guerras, crisis económicas, dictaduras, etc. Subrayando la capacidad de los eventos de ejercer una influencia sobre los distintos grupos que componen una sociedad, Grimaldi $(2009$, p. 5) evoca la dictadura como "elemento socializador de contexto", que cristaliza algunas actitudes políticas y marca varias generaciones de chilenos. Sin embargo, el régimen autoritario no es el único elemento contextual que hay que tomar en cuenta para entender la actualidad de las movilizaciones estudiantiles chilenas: todo lo contrario. El contexto postautoritario en el cual evolucionan los "hijos de la democracia" constituye una marca común de esta nueva generación que "no vivió en carne y huesos las prácticas de la dictadura y que tiene menos miedo de salir a la calle" (V.D., comunicación personal, enero de 2014) ${ }^{14}$. Más de la mitad de los estudiantes que hemos entrevistado nos han evocado este sentimiento de pertenencia generacional, algunos para subrayar el potencial movilizador de esta nueva generación, que "ha sabido aprender para organizarse mejor" (T.L., comunicación personal, febrero de 2014), otros para evocar el "proceso de aprendizaje de esta generación entre el 2006 y el 2011" (M.C., comunicación personal, febrero del 2014), otros finalmente asociándole un sentimiento de "deber y responsabilidad" (V.S., comunicación per-

\footnotetext{
14 Algunos de nuestros entrevistados pidieron explícitamente que no mencionáramos su nombre. Por razones de coherencia y para facilitar la lectura, decidimos mantener en el anonimato a los entrevistados citados en este artículo.
} 
sonal, abril del 2015). Muchas de las entrevistas que hemos llevado a cabo permiten observar que los primeros compromisos en la revuelta de los pingüinos contribuyeron a forjar disposiciones que los miembros de esta generación son capaces de reactivar en 2011. Al respecto, Muñoz Tamayo y Durán (2019) mencionan que la dinámica de las movilizaciones de 2011 se pueden explicar en parte por los "condicionantes generacionales" de sus protagonistas (p. 151). Dichos autores ponen de relieve el hecho de que los actores de esas protestas son jóvenes de entre 15 y 25 años, que realizan su socialización política en un contexto "alejado de las tensiones del periodo postransición" y "con la figura de Pinochet fuera de la agenda pública" (Muñoz Tamayo y Durán, 2019, p. 151). Se puede formular una hipótesis muy sólida respecto de una continuidad generacional entre los manifestantes de 2011 y de 2006, hasta los de 2001, según algunos autores (Thielemann, 2011; Donoso 2016). En el 2011 esta misma generación —“"más madura políticamente", según varios estudiantes que hemos entrevistado- se organiza nuevamente desde las universidades, en un contexto político muy distinto a las movilizaciones de 2006.

En efecto, la coalición de centroderecha, liderada por Sebastián Piñera, está al mando del país desde hace un año. Frente a gobiernos de centroizquierda, los movimientos sociales estaban confrontados a autoridades que habían luchado a favor de la democracia, y la llegada al poder de un gobierno de otra tendencia política representa un nuevo escenario político. Varios estudiantes nos evocaron este cambio de contexto como uno de los factores de explicación de las manifestaciones de 2011. Si el año 2010, marcado por el terremoto del 27 de febrero, no favorece la movilización, el 2011 se caracteriza desde sus inicios por un clima de agitación social — protestas en torno a la plataforma "Patagonia sin represas", intensificación de las manifestaciones mapuches, etc. Es en ese clima de agitación que la generación de los "hijos de la democracia", movilizada en 2006, está de nuevo en las calles. El nuevo contexto político, así como las deficiencias de la LGE del año 2008, favorecen la emergencia de movilizaciones masivas y 
su mantenimiento en el tiempo. Además, Barozet (2011b) subraya que se puede considerar estos eventos como "propios de un país cada vez más rico, pero en el que las desigualdades socioeconómicas son muy evidentes y no están frenadas por las políticas públicas"' (p. 129). Aparte de las demandas no satisfechas en 2006, las reivindicaciones del 2011 se centran en los mecanismos de acceso a la educación superior, la calidad de la enseñanza, y una transformación del sistema de financiamiento que permitiera alcanzar la gratuidad de la educación. Los estudiantes introducen también un discurso en torno a la lucha en contra de la herencia de la dictadura, no solo económica sino también política y social. Los estudiantes movilizados agregan progresivamente a sus reivindicaciones sectoriales demandas más generales: reformas fiscales, renacionalización del cobre, nueva Constitución. La crítica que se formula en 2011 sobrepasa claramente el estricto dominio de la educación para dirigirse al modelo chileno en su conjunto y su "consenso neoliberal", al origen de un sistema profundamente desigual (Cortés, 2015, p. 22).

Las movilizaciones más importantes empiezan a mediados de mayo, cuando la CONFECH convoca una de las primeras manifestaciones masivas del movimiento, frente al descontento creciente de los estudiantes hacia la política del gobierno en materia de educación. Siguen a un ritmo sostenido a lo largo del 2011, alcanzando niveles de participación inigualados desde el retorno a la democracia (Salazar, 2012). Varias encuestas revelan un apoyo masivo de la población a las demandas de trasformación formuladas por los estudiantes, mientras que la popularidad del gobierno cae ostensiblemente (Vera Gajardo, 2011; Cortés, 2015). Además, los estudiantes extienden el debate sobre la educación a una reflexión más general sobre los mecanismos institucionales y políticos al origen de la situación de la educación en Chile. Con frecuencia, los estudiantes denuncian la colusión entre varios miembros de la clase política y los consejos de administración de colegios y universidades, o su asociación con establecimientos privados. El cambio de Constitución se convierte en la piedra 
angular de las reivindicaciones estudiantiles, porque garantiza el papel subsidiario del Estado en materia de educación y consagra el principio de superioridad del mercado en este sector. Se expresaron las distintas demandas del movimiento estudiantil durante múltiples días de movilización, en los que se llevaron a cabo varias modalidades de acción, que examinaremos ahora.

\section{Repertorios: clivajes políticos y adaptabilidad estratégica}

En esta sección nos proponemos examinar los distintos tipos de acciones implementadas por los estudiantes, que componen lo que Tilly (1984) llama "repertorio de la acción colectiva" (p. 99). Esta noción designa el conjunto preexistente de formas de acción colectiva contenciosa, las "performances" (o representaciones) que los grupos movilizados tienen a su disposición en un momento dado para llevar a cabo una protesta. En el sentido propio, el término se refiere a una "recopilación metódica" (Goirand, 2010, p. 16), en la cual los elementos están organizados según un sistema que permite encontrarlos fácilmente. El repertorio constituye también el conjunto de las obras que un artista es capaz de interpretar. Aplicado a la acción colectiva, bajo esa doble acepción, la herramienta teórica del repertorio presenta una serie de ventajas analíticas. Primero, la metáfora artística permite subrayar el hecho de que la acción colectiva descansa sobre una serie de elementos aprendidos, memorizados, pero que puede también dejar sitio a la improvisación y la innovación. Entonces, la composición de un repertorio depende de los medios que los actores están dispuestos a movilizar, pero también del sistema político en el cual existe, entendido como contexto histórico y recurso de la movilización. La cuestión de la improvisación permite entrever la posibilidad de una transformación del repertorio, que es "gradual y traduce la transformación de los intereses, del entorno político, así como el cambio de posiciones políticas y sociales de los grupos contestatarios" (Goirand, 2010, p. 17). El análisis de 
esos elementos revela varias características del movimiento estudiantil: permite primero entender el proceso de toma de decisiones interno, revelando a la vez la capacidad de adaptación de los estudiantes al contexto político que les rodea. Dicho análisis revela también la diversidad de las organizaciones que componen el movimiento y las principales divergencias que lo animan. El tema del uso de la violencia, dejado de lado por muchos de los estudios sobre las movilizaciones estudiantiles, hará el objeto de una atención detallada al final de esta sección.

El elemento del repertorio de acción colectiva más usado por los estudiantes, especialmente en 2011, es sin ninguna duda la manifestación, conocida en Chile como "marcha". Es también el único elemento del repertorio cuya implementación se decide al nivel de las instancias de representación del movimiento en su conjunto, tales como la CONFECH o la ACES, cuyos miembros están a cargo de negociar la organización de los desfiles con las autoridades políticas y las fuerzas del orden. Modo de acción relativamente consensual dentro del movimiento, se implementa generalmente cuando se buscar apelar al nombre (Offerlé, 1998) y "demostrar la fuerza de los estudiantes" (entrevista con G.P., abril de 2015). Los textos de ley que rigen ese tipo de acción garantizan constitucionalmente el derecho a manifestar, sin autorización, siempre y cuando el acto sea pacífico y sin armas. Pero estos textos explican también que cualquier reunión en un lugar público tiene que ser notificada a las autoridades competentes por los menos dos días antes del evento, y que las fuerzas policiales podrán impedir o disolver cualquier manifestación que no respete este plazo de notificación. En Santiago de Chile las demandas se dirigen a la Intendencia de la Región Metropolitana, y se preparan las manifestaciones durante reuniones de trabajo en presencia de los organizadores del desfile, el responsable del orden público de la Intendencia y representantes de las fuerzas policiales. Si los textos de ley no indican que las autoridades pueden formalmente prohibir una manifestación, el margen de maniobra que tienen para restringir las marchas es la capacidad 
que les otorga el Decreto 1.086 para determinar el recorrido de los desfiles. Esta cuestión es una preocupación central, tanto para las autoridades, preocupadas por los posibles desmanes, como para los estudiantes, que buscan tener acceso a la principal arteria de la capital, la Avenida Bernardo O'Higgins, también conocida como "Alameda". En 2011, uno de los medios utilizados por la Intendencia de la Región Metropolitana para intentar frenar la contestación fue de restringir el acceso de los manifestantes a calles poco transitadas y alejadas de la Alameda. La composición de los cortejos es reveladora de la diversidad de los grupos y organizaciones que componen el movimiento. $\mathrm{Al}$ frente del desfile podemos encontrar a los organizadores de la manifestación, tales como los representantes de la CONFECH o de las organizaciones secundarias. En el resto del desfile, banderas con los colores de los colegios y de las universidades predominan sobre los estandartes de las juventudes partidarias, mientras que en algunas ocasiones se puede observar banderas roja y negro, símbolos de la izquierda revolucionaria. Las identidades colectivas que resaltan en esas manifestaciones son más universitarias que partidarias, sin que se desconozca la pertenencia política de algunos líderes del movimiento. Los estudiantes movilizados también importan eslóganes y modos de actuar del antidictadura, recorriendo elementos centrales del imaginario político colectivo chileno: se puede escuchar con frecuencia los manifestantes repetir "El pueblo, unido, jamás será vencido", o "El que no salta es facha", "El que no salta es Piñera"... Esta reproducción demuestra la existencia de un importante trabajo de filiación histórica realizado por los estudiantes. Es también frecuente que distintas bandas y orquestas toquen música, especialmente cumbia, generando a su alrededor pequeños grupos de gente que baila durante la marcha. Traïni (2008) subraya que los "dispositivos musicales" pueden tener papeles muy diferentes en la acción colectiva: permiten por ejemplo la expresión de emociones necesarias a la exaltación de un "nosotros" inclusivo, o a la modulación táctica de posturas afectivas apropiadas a la situación (p. 122). En el caso que nos interesa, 
el uso de la música en las manifestaciones contribuye a dar una dimensión festiva, hasta carnavalesca, y relajar el ambiente a veces saturado por los gases lacrimógenos.

El segundo medio de acción implementado por los estudiantes se conoce en Chile bajo el nombre de "toma", que constituye uno de los elementos centrales del repertorio de la acción colectiva chilena y que nace en los años 50. La toma, que consiste en esa época en la ocupación ilegal de terrenos para instalar campamentos, se generaliza en los años 1950-1960. Tal como lo explica el historiador Gabriel Salazar (2012), la ocupación de terrenos toma progresivamente una forma colectiva y planificada, destinada a controlar un espacio para construir una población. Ese modo de acción se extiende rápidamente a otros sectores de la sociedad en su movilización, en particular al sector estudiantil, que no duda en ocupar los edificios escolares desde mediados de los 60 (Salazar, 2012). Hoy en día, las tomas consisten en introducirse, de noche generalmente, en los establecimientos escolares para tomar el control e interrumpir el funcionamiento normal. El uso de ese método de acción, que permite inscribir la movilización en el tiempo, se decide al nivel local de cada colegio y universidad, y la decisión de reconducirlo en el tiempo se somete frecuentemente a votación, así como nos lo comentaron varios líderes estudiantiles que entrevistamos. Pancartas con las principales reivindicaciones y / o las razones de la acción están instaladas en la entrada de los edificios ocupados, cuyos accesos están bloqueados por sillas, mesas y cadenas metálicas. El carácter ilegal de ese modo de acción no frena para nada a los estudiantes, que lo usan mucho, especialmente en los colegios.

En el año 2006 los secundarios privilegiaron primero la movilización callejera, pero varios elementos les llevaron a cambiar de estrategia. Mientras que los principales medios de comunicación tienden a criminalizar el movimiento, las primeras manifestaciones son brutalmente reprimidas por las fuerzas del orden, y los desmanes provocados por grupos de encapuchados - jóvenes que actúan con la cara cubierta por un pañuelo o una camiseta- 
contribuyen a deslegitimar al movimiento (Bonner, 2014; Cortés, 2015). Las tomas se multiplican en una lógica de repliegue estratégico hacia el interior de los establecimientos escolares, lo que demuestra la capacidad de reacción y de adaptación de los secundarios. En un par de semanas estas prácticas se extienden por el territorio nacional y el repertorio de acción se vuelve estático, lo que permite reducir de forma considerable los enfrentamientos con la policía, contribuyendo a la vez a la visibilidad de las reivindicaciones secundarias. A partir de julio de 2011 se reproduce ese modo de acción después del llamado a la movilización realizado por la $\mathrm{CONFECH}$, afectando no solo a los colegios sino también a distintas universidades del país.

Junto al uso de elementos tradicionales del repertorio de la acción colectiva chilena, los estudiantes organizan también lo que denominan "actos culturales", designando así producciones artísticas o teatrales diversas. Dichos elementos aparecen en 2011, y el uso creciente de las redes sociales por los jóvenes chilenos facilita la organización de esos eventos, que nacen principalmente de iniciativas individuales masivamente transmitidas en la red. Mencionaremos algunos ejemplos, como las "1.800 horas alrededor de La Moneda", cuando los estudiantes de la Universidad de Chile realizaron una maratón en torno al palacio presidencial; el "Thriller por la educación", cuando tres mil jóvenes, disfrazados de zombis, reprodujeron de manera muy coordinada la coreografía de la canción de Michael Jackson; o la organización de "Flash mobs", tales como el denominado "Todos de vacaciones" en julio del 2011, cuando varios estudiantes, armados con toallas de playa y bloqueador solar, protestaron en contra de la decisión del ministro de Educación de adelantar la fecha de las vacaciones escolares, en pleno apogeo de las movilizaciones. El registro novador de esas acciones en el espacio contestatario chileno permitió la realización de distintos objetivos: desactivar el costo de cualquier intervención policial brutal frente a manifestantes disfrazados o en traje de baño, interpelar a las autoridades políticas gracias a la presencia masiva de los profesionales de los medios 
de comunicación, contrarrestando a la vez un contexto mediático normalmente desfavorable a la contestación (Bonner, 2014).

El carácter pacífico y casi festivo de estas manifestaciones no debe hacernos perder de vista las ocurrencias violentas y muy frecuentes al final de las marchas, a veces incluso cuando estas no han terminado, a solo algunos metros del lugar donde está instalado el escenario para el evento de cierre de la manifestación. Mientras que varios dirigentes estudiantiles o sindicales toman la palabra en dicho escenario, grupos de encapuchados atacan paradas de autobús, destruyen la señalética, o atacan a la policía estacionada algunos metros atrás con adoquines u objetos inflamables. Las manifestaciones acaban a veces con intensos enfrentamientos entre manifestantes y fuerzas del orden, frente a las cámaras de las grandes cadenas de televisión nacional que transmiten esos eventos en bucle, a veces omitiendo el resto de la movilización. Aunque los estudiantes prefieren recurrir a elementos del repertorio que valorizan la consigna o reivindican un carácter festivo y alegre, la violencia manifestante se impone con frecuencia al final de las marchas. Sabemos, por haber entrevistado a algunos, que miembros del movimiento estudiantil sí se encapucharon y participaron de enfrentamientos con Carabineros en las movilizaciones de $2011^{15}$. Sin embargo, encapuchados y modos de acción violentos son pocas veces objeto de un análisis detallado en los estudios sobre la protesta estudiantil en Chile. Muchas de las investigaciones sobre las movilizaciones de los estudiantes chilenos tienden a sólo constatar la existencia de enfrentamientos entre algunos manifestantes y las fuerzas del orden, sin interrogarse siquiera sobre la visión del movimiento al respecto, ni sobre la percepción de los actores que utilizan dichos modos de acción. Así, el trabajo de Donoso

15 Existe también la posibilidad, aunque no verificada, de que algunos de los encapuchados sean miembros de las fuerzas del orden, que se infiltran en las manifestaciones para provocar desmanes y justificar así la intervención policial durante las marchas. 
(2016), basado en unas cuarenta entrevistas realizadas con estudiantes secundarios y universitarios que participaron en distintas olas de movilizaciones, permite entender las estrategias del movimiento, así como sus modos de acción, y analizar algunas de sus divisiones. La cuestión de la violencia está mencionada en solamente dos ocasiones: observada, nunca está pensada como una posible estrategia reivindicada por algunas organizaciones estudiantiles (Donoso, 2016). Los mismos comentarios se pueden aplicar a las investigaciones de Vera Gajardo (2011) por ejemplo. No obstante, la confrontación con las fuerzas del orden constituye un parámetro central para entender el desarrollo de las movilizaciones de los estudiantes chilenos. En efecto, y como nos lo explicaron algunos dirigentes estudiantiles, optar por la toma o por un acto cultural, en vez de una marcha, se debe en cierta medida a la voluntad de evitar los enfrentamientos con la policía, que siguen siendo agotadores, que desalientan a los estudiantes más moderados y aparecen como contraproducentes por la imagen deslegitimante transmitida por los medios de comunicación. En ese sentido, los actos culturales pueden constituir una réplica táctica a los efectos producidos por esas imágenes de enfrentamiento.

La cuestión del uso de la violencia sigue siendo un tema muy espinoso al interior del movimiento, tema que se aborda con frecuencia en las reuniones de la $\mathrm{CONFECH}$, pero que jamás se ha zanjado de manera definitiva ${ }^{16}$. Las entrevistas que hemos llevado a cabo con el estudiantado chileno revelan la existencia de tres grandes tendencias respecto del tema. Primero, los que rechazan el uso de la violencia, sin expresar un discurso especialmente hostil hacia los de sus camaradas que

\footnotetext{
16 Cabe mencionar que la cuestión de la violencia es un tema en sí mismo, cuya complejidad no puede abarcarse en este artículo. Tendremos en cuenta la hipótesis que plantea que la violencia estructural es un componente central de la sociedad chilena, que se expresa de distintas maneras y da lugar a fenómenos como el de los encapuchados, que acaban siendo uno de los elementos característicos de las marchas estudiantiles.
} 
sí la utilizan. Encontramos ahí a miembros del "NAU" (Nueva Acción Universitaria), grupo político de centroizquierda creado en la Pontificia Universidad Católica de Santiago ${ }^{17}$, así como militantes de los partidos de la Nueva Mayoría ${ }^{18}$. Segundo, los que, con una visión pragmática de los modos de acción, consideran la violencia como una opción táctica útil en algunas circunstancias. Esta segunda tendencia aparece también como la más transversal, en la medida en que son estudiantes de grupos políticos muy distintos que demostraron cierto pragmatismo cuando les interrogábamos sobre el hecho de recurrir a la violencia. Si su uso nunca se presenta como el elemento central de la movilización, sí se considera en ciertas condiciones: cuando es "consensual y decidido por todos para presionar al gobierno" (V.S., comunicación personal, abril de 2015); en una "situación de defensa, para seguir con la toma y evitar el desalojo de un colegio" (D.V., comunicación personal, julio de 2016), o "para dañar la imagen pública del gobierno, hacer que luzca como incompetente, y generar cierto equilibrio en las negociaciones" (G.P., comunicación personal, abril de 2015). Encontramos finalmente a aquellos para quienes la violencia constituye un medio plenamente legítimo de la contestación. Más escasos y sobre todo más discretos al respecto son los estudiantes que reivindicaron abiertamente la legitimidad de recurrir a la violencia, no sin formular algunas reservas respecto de una posible extensión de su uso.

Esas divergencias acerca del tema espinoso de la violencia explican en gran parte la ausencia de estrategias claras en la CONFECH sobre la manera de enfrentar esos eventos cuando surgen en una manifestación ${ }^{19}$. En el año 2011, y frente a las di-

\footnotetext{
17 Que estará al mando de la Federación de Estudiantes de la Pontificia Universidad Católica (FEUC) durante varios periodos seguidos.

18 La Nueva Mayoría es el nombre tomado por la Concertación después de la incorporación del Partido Comunista en 2012-2013.

19 Para un análisis más detallado de los clivajes internos al movimiento estudiantil, véase Avendaño (2014).
} 
visiones del movimiento estudiantil, se instaló una especie de compromiso - en el sentido de la palabra inglesa compromiseen la CONFECH en torno a dos dinámicas. Se trata primero de un cierto acuerdo, tácito, en torno a la utilidad de la violencia como posible herramienta de comunicación para atraer la atención de la prensa, tanto nacional como internacional, sobre los eventos. No perdamos de vista que los enfrentamientos entre manifestantes y fuerzas del orden se acompañaron también de denuncias crecientes respecto de abusos policiales y prácticas de la policía militarizada chilena. Fue en torno a esa cuestión, "denunciar la represión" (P.G., comunicación personal, mayo de 2015), que se generó un segundo elemento de acuerdo que permitiera sobrepasar las divisiones internas en cuanto al uso de la violencia. Así, las divergencias al respecto fueron en parte eliminadas por un discurso consensual sobre la violencia policial y, más generalmente, sobre la violencia del Estado chileno, que "nos niega el derecho a educarnos dignamente, y luego a manifestarnos para demandar una mejor educación" (G.J., comunicación personal, enero de 2014).

Para concluir con el análisis de los elementos que componen el repertorio de la acción colectiva de los estudiantes chilenos, queda por examinar la reconversión de algunos dirigentes estudiantiles a la política institucional, así como el impacto de las movilizaciones de 2011 sobre el sistema partidario chileno.

\section{Hacia el parlamento y las instituciones públicas: trayectorias de reconversión}

Centraremos ahora nuestra atención sobre el surgimiento de una nueva organización partidaria que procede directamente de las movilizaciones, así como en la entrada en la política institucional - en el parlamento o en distintos servicios gubernamentales-de varias figuras del movimiento estudiantil. 
En enero de 2012, se crea el partido Revolución Democrática (RD), como "movimiento político que nace en el contexto de las movilizaciones sociales de 2011"20. En noviembre de 2013, cuatro antiguos líderes del movimiento son electos diputados: Camila Vallejo y Karol Cariola, ambas del Partido Comunista, Giorgio Jackson, para Revolución Democrática, y Gabriel Boric, figura de liderazgo de la Izquierda Autónoma (IA) que se presenta como independiente (IA) ${ }^{21}$. Cabe subrayar que la IA es una organización que deriva del movimiento SurDa y que supo aprovechar el capital político heredado de ella (Avendaño, 2014). En noviembre de 2017 RD elige diez diputados al parlamento, dos IA, y los líderes de 2011 son reelectos en sus puestos. Como podemos ver, en muy pocos años una parte del liderazgo estudiantil consolida su legitimidad electoral, a la vez que la recién creada RD asienta su presencia en las federaciones universitarias y en las manifestaciones públicas.

En su sitio web, RD se define como un movimiento político que "aspira a convertirse en un actor relevante de la política nacional"22. Según nos indicaron los miembros fundadores, así como militantes de RD que hemos podido entrevistar, fueron Giorgio Jackson y Miguel Crispi los que impulsaron la creación del movimiento, pensado como continuidad del NAU y de las acciones llevadas a cabo en la federación de la Pontificia Universidad Católica de Santiago (G. J.; M. C.; N. H.; comunicación personal, años 2014 y 2015). Aunque el sector dirigente original proviene de dicha federación, este partido acabó reuniendo a militantes provenientes de varias tradiciones políticas —antiguos

\footnotetext{
20 Ver «Qué es RD». URL: http://www.revoluciondemocratica.cl/que-es-rd/.

21 Oficialmente, Giorgio Jackson se presenta como independiente. Respecto a eso, la ley indica que el candidato debe contar con el apoyo "ciudadano", mediante firmas, de por lo menos un $0,5 \%$ de los votantes de las últimas elecciones parlamentarias en la circunscripción en la cual el candidato quiere presentarse. Gabriel Boric tuvo que seguir el mismo procedimiento. Sobre este tema, ver http://www.bcn.cl/leyfacil/ recurso/elecciones-parlamentarias.

22 «Qué es RD». URL: http://www.revoluciondemocratica.cl/que-es-rd/.
} 
socialistas, por ejemplo. Según las cifras del SERVEL — servicio electoral chileno-, actualizadas en abril de 2017, más de diez mil personas están afiliadas actualmente a RD, y cerca de 2.800 peticiones están esperando una confirmación (Servel, 2017). Conocer a varios militantes del joven partido nos permitió interrogarles sobre las razones de tanto entusiasmo, y muchas respuestas ponen de relieve que RD puede aparecer como un espacio ideal de formación y aprendizaje político, más flexible y menos estructurado que las organizaciones partidarias tradicionales, un "espacio más atractivo que el PS o el PPD para aprender a militar" según C.M. La proximidad y las relaciones construidas durante la movilización estudiantil son algunos de los motivos de la creación de RD. Son esos mismos elementos, esas experiencias compartidas en distintos espacios sociales, los que permitirán el acercamiento entre distintas organizaciones provenientes del movimiento estudiantil en torno a la plataforma electoral que se dará a conocer como "Frente Amplio"23. Beatriz Sánchez, candidata de la coalición, termina tercera en la primera vuelta de las elecciones presidenciales de 2017, y veinte diputados del Frente entran al Congreso de los diputados chilenos, después de solo unos meses de existencia ${ }^{24}$.

Junto a la creación de nuevas organizaciones políticas, los dirigentes estudiantiles que siguieron su acción política después de su participación activa en las movilizaciones del 2011 lo hicieron mediante la lucha electoral y/o la acción institucional. En ese sentido, Dechézelles y Luck (2011) subrayan cuan permeable y borrosa está la frontera entre acción colectiva y sistema político. Según ellos, existe un continuum entre política institucional y no institucional, ya que las interacciones entre ambas esferas son constantes y pasar de una a otra es relativamente fácil (Deché-

\footnotetext{
23 Oficialmente creado en septiembre de 2017, el Frente Amplio agrupa varias organizaciones políticas: RD, IA y otras formaciones más pequeñas, tales como el Partido Humanista o el Partido Ecologista Verde.

24 "Historia de los partidos", Biblioteca Nacional del Congreso. URL: https:// www.bcn.cl/historiapolitica/partidos_politicos/wiki/Frente_Amplio.
} 
zelles y Luck, 2011, p. 21). En el caso que nos interesa, el cambio de esferas de acción fue bastante rápido, ya que solo dos años después del inicio de las movilizaciones masivas de 2011, cuatro de las figuras más emblemáticas del movimiento fueron electas en el Congreso. Respecto de eso, Donoso (2016) nos habla de la puesta en marcha de una "estrategia de insider" por parte del movimiento, que consiste en comprometerse en la arena institucional y electoral para promover las demandas del movimiento. Mathieu (2011) explica que la voluntad de "confrontarse a cierta realidad de la acción política" y de renunciar a una postura de contestación, así como el deseo de pasar de preocupaciones más restringidas a intereses más generales, son las motivaciones más invocadas para justificar tales reconversiones. Las entrevistas que hemos llevado a cabo con algunos de esos conversos a la política institucional nos permiten observar que se evocan razones similares para explicar esos cambios de trayectoria: "tenía ganas de seguir actuando, pero desde otros espacios"; "quería continuar mi acción política, pero desde lo institucional", o "pensaba que podía transformar el sistema desde dentro después de haber luchado en la calle", son algunos de los motivos que nos evocaron las y los estudiantes entrevistados. Tal como lo mencionamos, varios de los líderes de las movilizaciones de 2011 se presentaron a las elecciones parlamentarias en 2013, algunos fueron reelectos -Jackson, Boric, Vallejo por ejemplo-, y algunos miembros de RD y de IA entraron al Congreso después de la votación de 2017.

Para explicar esas reconversiones, podemos terminar nuestro análisis mencionando el concepto de "capital militante", desarrollado por Poupeau y Matonti (2004). Según esos autores, el capital militante se distingue del capital político, "incorporado bajo la forma de técnicas, de disposiciones a actuar, intervenir o simplemente obedecer"; designa un conjunto de "saberes y saber-ser movilizables durante acciones colectivas o entra-partidistas" (Poupeau y Matonti, 2004, p. 8). Esas herramientas también son exportables, por lo tanto, pueden facilitar ciertas reconversiones: la capacidad de convocar una manifestación, de redactar 
un comunicado público, de formular demandas de reforma estructuradas o críticas hacia ciertas políticas públicas, son de los aprendizajes que pueden ser usados tanto en la esfera de los movimientos sociales como en la de la política institucional.

Podemos encontrar una versión un poco distinta de esa "estrategia de insider" (Donoso, 2016) entre los dirigentes estudiantiles que deciden participar en las distintas administraciones del segundo gobierno de Michelle Bachelet (2014-2018), buscando influenciar la concepción y realización de ciertas reformas, especialmente las vinculadas a las reivindicaciones formuladas en las movilizaciones de 2011. Por ejemplo, Miguel Crispi, miembro fundador de RD, será un cercano consejero del ministro de Educación, entre marzo de 2014 y mayo de 2016; Gonzalo Muñoz, miembro activo de RD y responsable de las cuestiones de educación para ese partido, estará a cargo de la nueva “División General de Educación", organismo a cargo de poner en pie la reforma de la educación pública del Ministerio de Educación chileno. Pedro Glatz, vicepresidente de la FEUC junto a Giorgio Jackson, obtendrá un puesto de responsable en la Dirección de Asuntos Sociales, mientras que Camilo Ballesteros, antiguo presidente de la FEUSACH, se encontrará a la cabeza de ese organismo público.

Así, el compromiso en la lucha electoral o en la acción institucional constituye el último elemento del repertorio de acción de ese movimiento heterogéneo, que lucha desde finales de los 90 en contra de la mercantilización del sistema educativo chileno.

\section{A modo de conclusión}

Como pudimos observar, el modelo educativo chileno implementado durante la dictadura y mantenido, aunque reformado, por los distintos gobiernos democráticos, fue cuestionado profundamente durante dos olas de movilización masivas protagonizadas por los estudiantes secundarios y de educación superior en distintas ciudades del país. En un contexto político e institucional 
poco propicio a la movilización, la renovación de las generaciones y de los comportamientos contenciosos, así como los procesos de aprendizaje durante la movilización, aparecen como elementos centrales para entender la Revolución pingüina y la Primavera de Chile. Construida en torno a un discurso de lucha contra la herencia económica y política de la dictadura, la contestación que se inicia en 2006 y prosigue en 2011 consigue generar movilizaciones muy diversas, a menudo masivas. Dicha masividad pone de relieve el afán de participación de una nueva generación, y la diversidad de modos de acción del movimiento estudiantil es reveladora de las capacidades estratégicas, movilizadoras y de adaptación de los jóvenes chilenos frente a las tácticas de la policía, de las autoridades y de los medios de comunicación. Revelan también la riqueza, creatividad y heterogeneidad interna del movimiento, en particular las frecuentes confrontaciones entre manifestantes y policía en el contexto de numerosas manifestaciones estudiantiles. Pudimos observar cómo los estudiantes intentaron sobrepasar sus divisiones internas, especialmente respecto del uso de la violencia, y también analizar la manera con la que algunos dirigentes del movimiento decidieron transferir sus competencias hacia el sistema político e institucional.

\section{Referencias}

Águila, E. (2010). Veinte años de Concertación en educación: avances y límites de una reforma. En Ensignia, J. (Ed.), Chile en la Concertación (1990-2010). Una mirada crítica, balance y perspectivas. TOMO II (pp. 215-235). Santiago de Chile: Friedrich Ebert Stiftung.

Assael, J., Cornejo, R., González, J., Redondo, J., Sánchez, R. y Sobarz, M. (2011). La empresa educativa chilena. Revista Educación E Sociedad, 32(115), 305-322.

Avendaño, O. (2014). Fracturas y representación política en el movimiento estudiantil. Chile 2011. Última Década, (41), 41-68.

Azócar, C. (2013). El movimiento estudiantil chileno: balances y perspectivas. El giro sociopolítico en el nuevo escenario electoral. Anuari del conflicte social, Universidad de Barcelona. 
Barozet, E. (2011a). De la démobilisation au réinvestissement «local». Mouvements sociaux locaux et territoires au Chili. Cahier des Amériques Latines, (66), 73-89.

Barozet, E. (2011b). Brève. Problèmes d'Amérique latine, (82), 129-131.

Braghetto, M. (2013). El movimiento universitario y las transformaciones de la educación superior en el Chile neoliberal. Revista Izquierdas, (16), 55-74.

Bonner, M. (2014). Policing protest in Argentina and Chile. Boulder, Colorado: Lynne Rienner Publishers.

Brunner, J. J. (2009). Mercados, instituciones y políticas en la educación superior chilena. Temas: Cultura, Ideología, Sociedad, (57), 52-59.

Brunner, J. J. (2011). Visión histórica de la evolución del sistema de educación superior chileno: hitos desde 1967 a la fecha. En M. Jiménez y F. Durán (Eds.), Un recorrido por la Historia Reciente de la Educación Superior Chilena (pp. 23-39). Santiago de Chile: Aequalis-Foro de la Educación Superior.

Cortés, A. (2015). Chile en la encrucijada de un nuevo ciclo. Cadernos de trabalho Netsal, 3(7), 43 p.

Dabène, O. (2008). Enclaves autoritaires en démocratie: perspectives latino-américaines. En O. Dabène, V. Geisser y G. Massardier (dir.), Autoritarismes démocratiques et démocraties autoritaires au XXIe siècle. Convergences Nord-Sud (pp. 89-113). París: La Découverte.

Dechézelles, S. y Luck, S. (2011). Introduction. Mondes mouvementistes et partisans: points de contact, passerelles et pistes d'exploration. En S. Dechézelles y S. Luck S. (Dirs.), Voix de la rue ou voie des urnes? (pp. 9-28). Rennes: Presses Universitaries de Rennes.

Donoso, S. (2016). "Outsider" and "insider" strategies: Chile's student movement, 1990-2014. En S. Donoso y M. Von Bulöw, Social Movements in Chile. Organization, trajectories and political consequences. Nueva York: Palgrave-MacMillan.

Doran, M-C. (2016). Le réveil démocratique du Chili. Une histoire politique de l'exigence de justice (1990-2016). París: Khartala. .

Garretón, M. (1989). La posibilidad democrática en Chile. Santiago de Chile: FLASCO, Cuadernos de Difusión.

Garretón, M. y Garretón, R. (2010). La democracia incompleta en Chile: 
De la calle al parlamento: trayectorias y repertorios de una generación de estudiantes. Chile, 2006-2017 -

la realidad tras los rankings internacionales. Revista de Ciencia Política, 30(1), 115-148.

Goirand, C. (2010). Mobilisations et répertoires d'action collective en Amérique Latine. Revue Internationale de Politique Comparée, 17(2), 7-27.

Grimaldi, D. (2009). Generaciones políticas del socialismo democrático en Chile. Working Paper del Congreso IPSA 2009.

Huneeus, C. (2014). La democracia semisoberana. Chile después de Pinochet. Santiago de Chile: Taurus.

Ihl, O. (2002). Socialisation et évènements politiques. Revue Française de Science Politique, 52(2/3), 125-144.

Mannheim, K. (2011). Le problème des générations. Armand Colin, coll. «Hors Collection», (1 $1^{\text {a }}$ edición: 1990).

Mathieu, L. (2011). S'engager dans un parti et/ou dans un mouvement social: tensions et reconversions militantes. En S. Dechézelles y S. Luck (Dirs.), Voix de la rue ou voie des urnes? (pp. 149-163). Presses universitaires de Rennes.

Matonti, F. y Poupeau, F. (2004). Le capital militant. Essai de définition. Actes de la Recherche en Sciences Sociales, (155), 4-11.

Moulián, T. (1997). Chile, anatomía de un mito. Santiago de Chile: LOM.

Muñoz, G. y Raczynski, D. (2007). Reforma educacional chilena: el difícil equilibrio entre la macro y la micro política. Serie Estudios Socioeconómicos, (31).

Muñoz Tamayo, V. y Durán, C. (2019). Los jóvenes, la política y los movimientos estudiantiles en el Chile reciente. Ciclos sociopolíticos entre 1967 y 2017. Izquierdas, (45), 129-159.

Offerlé M. (1998). Sociologie des groupes d'intérêt. París: Monchrestien.

Salazar, G. (2012). Movimientos sociales en Chile. Trayectoria histórica y proyección política. Santiago de Chile: Uqbar Editores.

Servel (2017). Consulta de Afiliación y Certificado de Afiliación Política. Santiago de Chile: Servicio Electoral de Chile. Recuperado de https://www.servel.cl/wp-content/uploads/2017/04/Ratificaciones_mas_Afiliaciones_por_Partidos_11-04-2017.pdf.

Thielemann, L. (2011). Para una periodificación del movimiento estudiantil de la transición (1987-2011). Revista Pretérito Imperfecto, 2 .

Tilly, C. (1984). Les origines du répertoire d'action collective contem- 
poraine en France et en Grande Bretagne. Vingtième siècle - Revue d'Histoire, 4, 89-108.

Traïni, C. (2008). La musique en colère. París : Presses de Sciences Po.

Vera Gajardo, S. (2011). El resplandor de las mayorías y la dilatación de un doble conflicto: el movimiento estudiantil en Chile en el 2011. Anuari del Conflicte Social 2011, Universidad de Barcelona, 286-309. 